\title{
NOUVELle
}

\section{L'IL-23R, un nouveau gène de susceptibilité dans les maladies inflammatoires chroniques intestinales}

Laurent Peyrin-Biroulet, Erika Parmentier-Decrucq, Julien Branche, Pierre Desreumaux

\author{
Inserm U795 (ex EAl14), Centre de recherche \\ sur les maladies inflammatoires chroniques intestinales, \\ Hôpital Swynghedauw, rue A. Verhaeghe, \\ 59037 Lille Cedex, France. \\ pdesreumaux@hotmail.com
}

> La maladie de Crohn (MC) et la rectocolite hémorragique $(\mathrm{RCH})$ sont des maladies inflammatoires chroniques de l'intestin $(\mathrm{MICl})$ caractérisées par une anomalie de régulation de la réponse immunitaire muqueuse dirigée contre des éléments de la flore intestinale. Ces pathologies surviennent chez des patients génétiquement prédisposés.

\section{$\mathrm{MICl}$ et facteurs génétiques}

L'hypothèse génétique dans les $\mathrm{MICl}$ repose sur un faisceau d'arguments. Le premier est l'existence de formes familiales. La fréquence des formes familiales de $M C$ varie de $8 \%$ à $40 \%$ selon les études. Certaines familles exceptionnelles comportent un grand nombre de sujets atteints.
Le risque relatif (RR) de $\mathrm{MICl}$ pour les apparentés peut être calculé en faisant le rapport entre la prévalence de la maladie estimée dans la population des parents de sujets atteints et la prévalence dans la population générale. Le RR d'être atteint de $M C$ pour un parent au premier degré de sujet atteint de $M C$ est de 10 et le RR de développer une RCH pour un parent de premier degré de sujet atteint de RCH est de 8. Le RR de développer une RCH lorsqu'un parent au premier degré est atteint de $M C$ est lui aussi élevé $(3,85)$ alors que le risque inverse n'est pas significativement différent de celui qui prévaut dans la population générale [1]. Ainsi, une histoire familiale de $\mathrm{MICl}$ est le premier facteur de risque de développer une $\mathrm{MICl}$.
Le second argument est le fort taux de concordance chez les jumeaux homozygotes. L'étude de référence est une étude suédoise dans laquelle un croisement des registres de jumeaux et des registres de $\mathrm{MICl}$ a été réalisé, dans une population géographiquement définie [2]. Quarante-quatre paires de jumeaux dont un au moins était atteint de $M C$ et 36 paires dont un au moins était atteint de RCH ont été étudiées. Pour la MC, le taux de concordance chez les monozygotes $(58,3 \%)$ était significativement supérieur au taux observé chez les dizygotes $(3,9 \%$ ) (chiffre équivalent au risque relatif observé chez les parents au premier degré). Les taux de concordance étaient dans les deux cas supérieurs dans

\begin{tabular}{|c|c|c|c|c|}
\hline Locus & Gène & Chromosome & $\begin{array}{c}\text { Association à MC } \\
\text { ou RCH }\end{array}$ & Fonction de la protéine \\
\hline $\begin{array}{c}\text { IBDI } \\
\text { (inflammatory } \\
\text { bowel disease 1) }\end{array}$ & CARD15/NOD2 & 16 & MC & $\begin{array}{l}\text { Récepteur cytosolique des bactéries intra- } \\
\text { cellulaires (motif reconnu = muramyl dipeptide) }\end{array}$ \\
\hline \multirow[t]{4}{*}{ IBD5 } & $S L C 22 A 4 / 5$ & 5 & MC ? & OCTN1/2 (transporteurs de cations organiques) \\
\hline & DLG5 & 10 & MC? & Renouvellement épithélium \\
\hline & CARD4/NODI & 7 & MC? & $\begin{array}{l}\text { Récepteur cytosolique aux bactéries intra- } \\
\text { cellulaires (motif reconnu = i-EDAP) }\end{array}$ \\
\hline & $\begin{array}{l}\text { CARD8/TUCAN/ } \\
\text { CARDINAL }\end{array}$ & 19 & MC? & $\begin{array}{l}\text { Inhibiteur de la voie NF-kB et rôle anti- } \\
\text { apoptotique }\end{array}$ \\
\hline \multirow[t]{2}{*}{ IBD3 } & & 6 & $M C ?$ & Système HLA \\
\hline & $I L-23 R$ & 1 & $M C$ et $\mathrm{RCH}$ & $\begin{array}{l}\text { Récepteur de l'IL-23, défense contre bactéries } \\
\text { pathogènes? }\end{array}$ \\
\hline
\end{tabular}

Tableau I. Principaux gènes de susceptibilité aux MICI. MC : maladie de Crohn ; RCH : rectocolite hémorragique. 
la MC aux taux de concordance observés pour la RCH : dans cette maladie, il était de $6,3 \%$ chez les monozygotes, ce qui n'est pas significativement supérieur au taux observé chez les dizygotes (0\%). Une seule paire mixte MC-RCH a été jusqu'à maintenant observée [2]. Ces résultats sont en faveur d'une composante génétique forte mais non exclusive (car la concordance devrait être dans ce cas de $100 \%$ ) intervenant dans la physiopathologie de la MC contrairement à la RCH. La concordance incomplète observée entre jumeaux monozygotes confirme que les $\mathrm{MICl}$ ne sont pas des maladies à transmission mendélienne simple, mais elle ne permet pas de définir un modèle de transmission.

Plus récemment en 2001 , I'identification par deux équipes indépendantes de NOD2/CARD15 (codant pour une protéine intracellulaire contenant un domaine $\mathrm{N}$-terminal caspase recruitment, CARD) comme gène de susceptibilité à la $M C$ avait permis de souligner le rôle prépondérant des facteurs génétiques dans la pathogénie de cette maladie [3, 4]. D'autres gènes comme DLG5 (équivalent humain de discs, large homolog 5 (Drosophila) et SLC22A4/5) pourraient être associés à la MC [5]. Aujourd'hui, une

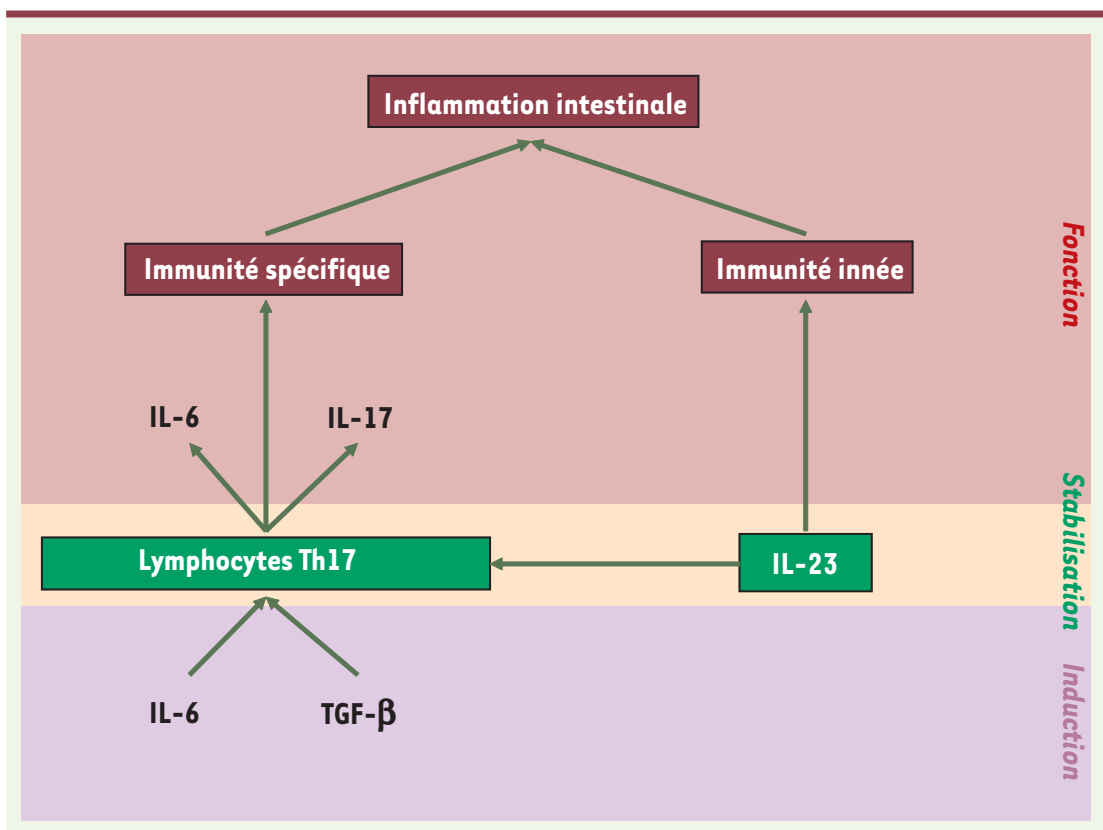

Figure 1. Rôle de l'IL-23 dans la régulation de l'inflammation intestinale.

équipe américaine a mis en évidence un nouveau gène qui pourrait protéger contre le développement des $\mathrm{MICl}$, situé sur le chromosome $1 \mathrm{p} 31$ et codant pour le récepteur de l'interleukine-23 (IL-23R) [6]. Les principaux gènes de susceptibilité aux $\mathrm{MICl}$ sont repris dans le Tableau I.

\section{IL-23R, un nouveau gène de susceptibilité dans les $\mathrm{MICl}$}

Cho et al. ont tout d'abord analysé 547 malades atteints d'une MC iléale et 548 sujets contrôles d'origine européenne, non juifs. Les auteurs ont choisi de restreindre leur analyse aux atteintes iléales de la MC afin de limiter l'hétérogénéité clinique et génétique des patients. Cette étude a mis en évidence une association inverse entre un allèle rare de l'IL23R et le risque de $M C$ à localisation iléale. Ainsi, le polymorphisme codant (rsl1209026, c.1142G>A, p.Arg381GIn) pour un acide aminé situé dans la partie intra-cytoplasmique de I'IL23R est moins fréquemment exprimé chez les patients non juifs ayant une MC à localisation iléale que chez les sujets témoins $(1,9 \%$ vs $7,0 \% ; O R=0,26 ; \mathrm{IC}$ à $95 \%: 0,15-0,43)$ ( $p$ corrigé $=1,56 \times 10^{-3}$ ). II existait également une association entre le risque de $M C$ iléale et un polymorphisme plus fréquent correspondant à 9 autres marqueurs non codants localisés soit au sein du gène de l'IL23R, soit entre le gène de I'IL-23R et celui de I'ILI2RB2 (interleukin 12 receptor, beta-2 chain).

Ces résultats ont été confirmés dans une cohorte indépendante de patients d'origine juive atteints de MC iléale, mais aussi dans 883 familles dans lesquelles les parents et leurs enfants étaient atteints de maladies inflammatoires chroniques de l'intestin (MC, RCH, ou colite d'origine indéterminée). Les résultats provenant de ces études familiales ont également montré une association entre IL23R et RCH, mais seulement chez les sujets non juifs.

\section{Quelle est la signification}

fonctionnelle du polymorphisme génétique de l'IL-23R?

À l'issue du travail de Cho et al. [6], une question vient tout naturellement à l'esprit: quelle est la fonction de ce récepteur? En d'autres termes, quel rôle pourrait jouer cette protéine dans la pathogénie des $\mathrm{MICl}$ ? Personne ne peut répondre aujourd'hui clairement à cette question. Les fonctions multiples et redondantes des cytokines et de leurs récepteurs permettent toujours d'envisager une fonction rationnelle protectrice ou prédisposante de ces médiateurs dans la physiopathologie des $\mathrm{MICl}$.

Classiquement, les lésions chroniques de MC sont associées à une augmentation de la synthèse d'IL-12 et d'IL-23, cytokines produites notamment par les macrophages de la lamina propria et orientant la réponse immunitaire vers un profil de cytokines Thl, caractérisé par une forte production d'IFN $\gamma$ (interféron $\gamma$ ). Plus récemment, une nouvelle famille de lymphocytes T appelée Th17, induits par I'IL-6 et le TGF- $\beta$ (transforming growth factor $\beta$ ), stabilisés par l'IL-23, et caractérisés par leur capacité à produire de l'IL-6 et de l'IL-17 a été mise en évidence au sein du système immunitaire muqueux intestinal $[7,8]$ (Figure 1). Ces lymphocytes Th17 et cette production d'IL-23 jouent un rôle important dans l'immunité muqueuse 
spécifique et innée, pouvant être à l'origine d'une susceptibilité aux infections bactériennes [9-11] et du déclenchement de mécanismes inflammatoires intestinaux, dépendants ou non de la présence de lymphocytes $T$ ou $B[7,8]$.

Ainsi, si l'on considère l'allèle rare $d u$ polymorphisme de I'IL-23R codant pour l'arginine en positon 381 (Arg381) dans la partie très conservée intracytoplasmique du récepteur, cette modification pourrait augmenter la fonction de l'IL-23 et conférer à la muqueuse iléale une meilleure résistance aux agressions bactériennes, protégeant contre la survenue de la MC. Le rôle de I'IL-23R au cours de la RCH, pathologie classiquement associée à un profil Th2, reste plus énigmatique.

\section{Conclusions}

L'allèle rare du polymorphisme codant pour l'Arg381GIn de I'IL-23R est inversement associé au risque de $M C$ iléale, familiale ou non, survenant chez des patients juifs ou non juifs, mais également dans les formes familiales de RCH survenant chez des patients non juifs. Cet allèle rare serait protecteur vis-à-vis de la MC, mais inversement, le polymorphisme de l'IL-23R pourrait prédisposer à la MC si l'on considère l'allèle fréquent.

La considération de travaux dogmatiques associant des pathologies chroniques à des profils de cytokines régulatrices (Th1, Th2, Th3...) dont l'existence chez l'homme est remise en doute ne facilite pas l'interprétation de ces résultats sur un plan fonctionnel. $\diamond$

IL-23R, a novel susceptibility gene

for inflammatory bowel disease

\section{RÉFÉRENCES}

1. Halme L, Paavola-Sakki P, Turunen U, et al. Family and twin studies in inflammatory bowel disease. World J Gastroenterol $2006 ; 12$ : 3668-72.

2. Tysk C, Lindberg $\varepsilon$, Jarnerot $G$, Floderus-Myrhed B. Ulcerative colitis and Crohn's disease in an unselected population of monozygotic and dizygotic twins. A study of heritability and the influence of smoking. Gut $1988 ; 29: 990-6$.
3. Hugot JP, Chamaillard M, Zouali $H$, et al. Association of NOD2 leucine-rich repeat variants with susceptibility to Crohn's disease. Nature 2001 ; 411 : 599-603.

4. Ogura Y, Bonen DK, Inohara N, et al. A frameshift mutation in NOD2 associated with susceptibility to Crohn's disease. Nature $2001 ; 411$ : 603-6.

5. Chamaillard M, lacob R, Desreumaux P, Colombel JF. Advances and perspectives in the genetics of inflammatory bowel diseases. Clin Gastroenterol Hepatol 2006 ; $4: 143-51$.

6. Duerr RH, Taylor KD, Brant SR, et al. A genome-wide association study identifies IL23R as an inflammatory bowel disease gene. Science 2006 ; 314 : 1461-3.

7. Hue S, Ahern P, Buonocore S, et al. Interleukin23 drives innate and $T$ cell-mediated intestinal inflammation. J Exp Med 2006 ; 203 : 2473-83.

8. Yen D, Cheung J, Scheerens H, et al. IL-23 is essential for $\mathrm{T}$ cell-mediated colitis and promotes inflammation via IL-17 and IL-6.J Clin Invest 2006 ; 116 : 1310-6.

9. Mangan PR, Harrington LE, O'Quinn DB, et al. Transforming growth factor-beta induces development of the T(H) 17 lineage. Nature 2006 ; 441 : 231-4.

10. Verreck FA, de Boer T, Langenberg DM, et al. Human IL-23-producing type 1 macrophages promote but IL10 -producing type 2 macrophages subvert immunity to (myco)bacteria. Proc Natl Acad Sci USA 2004 ; $101: 4560-5$

11. Cooper AM, Kipnis A, Turner J, et al. Mice lacking bioactive IL-12 can generate protective, antigenspecific cellular responses to mycobacterial infection only if the IL-12 p40 subunit is present. J Immunol $2002 ; 168: 1322-7$.

\section{NOUVELle}

\section{(1) \\ Comment le nez se connecte au cerveau}

Aurélie Mouret, Pierre-Marie Lledo
Unité « Perception et Mémoire », Institut

Pasteur, CNRS URA2182,

25, rue du Docteur Roux,

75724 Paris Cedex 15, France. pmlledo@pasteur.fr

le champ de l'olfaction, nos connaissances sur l'odorat n'ont cessé de s'accroître. Aujourd'hui, ce sens mystérieux livre peu à peu ses secrets et l'opinion publique est fascinée par la découverte du pouvoir des odeurs. Limité jusqu'alors aux mondes des poètes, gastronomes, œnologues et autres parfumeurs, ce sujet d'étude attire désormais l'attention des scientifiques contemporains. Aussi connaissons-nous assez bien les premières étapes qui participent à la perception et à la reconnaissance des molécules odorantes. Nous savons que la sélection des aliments, la détection des 